\title{
Genetic characterization of a reptilian calicivirus (Cro1)
}

\author{
Carlos Sandoval-Jaime ${ }^{1}$, Gabriel I Parra', Alvin W Smith ${ }^{2}$ Kim Y Green ${ }^{1}$ and Stanislav V Sosnovtsev ${ }^{1 *}$
}

\begin{abstract}
Background: Vesiviruses in the family Caliciviridae infect a broad range of animal hosts including mammals, birds, fish, amphibians and reptiles. The vesivirus Cro1 strains were isolated from diseased snakes in the San Diego zoo in 1978 and reported as the first caliciviruses found in reptiles. The goal of this study was to characterize the Cro 1 strain 7800321 that was isolated in cell culture from a rock rattlesnake (Crotalus lepidus) in the original outbreak.

Results: We re-amplified the original virus stock in Vero cells, and determined its full-length genome sequence. The Cro1 genome is 8296 nucleotides (nt) in length and has a typical vesivirus organization, with three open reading frames (ORF), ORF1 (5643 nt), ORF2 (2121 nt), and ORF3 (348 nt) encoding a nonstructural polyprotein, the major capsid protein precursor, and a minor structural protein, respectively. Phylogenetic analysis of the full-length genome sequence revealed that the Cro1 virus clustered most closely with the VESV species of the genus Vesivirus, but was genetically distinct (82-83\% identities with closest strains).
\end{abstract}

Conclusions: This is the first description of a full-length genome sequence from a reptile calicivirus (Cro1). The availability of the Cro1 genome sequence should facilitate investigation of the molecular mechanisms involved in Crol virus evolution and host range.

Keywords: Reptile calicivirus, Cro1, Complete genome, Vesivirus phylogeny

\section{Background}

The family Caliciviridae is a large group of small, nonenveloped RNA viruses that includes important human and animal pathogens [1]. The family is comprised of five genera: Lagovirus, Vesivirus, Nebovirus, Sapovirus and Norovirus [2] and two new genera have been proposed [3]. Despite marked genetic and antigenic diversity, caliciviruses share several common features. All have icosahedral virions with a protein shell containing 180 copies of a major capsid protein, VP1 [4]. The virions carry a positive-sense single-stranded RNA genome of approximately 6.4 to $8.5 \mathrm{~kb}$ in length. The 3 '-end of the calicivirus RNA is polyadenylated, and the 5 '-end is covalently linked to a small protein encoded by the virus genome, VPg [5]. Calicivirus RNA genomes share similar organization; they are comprised either of two or three ORFs. The large ORF1 encodes the virus nonstructural proteins and is expressed from the genomic RNA template. In the genomes of sapo-, lago-, and neboviruses, the nonstructural

\footnotetext{
* Correspondence: ss216m@nih.gov

${ }^{1}$ Caliciviruses Section/LID/NIAID/NIH, Bethesda, MD 20892, USA

Full list of author information is available at the end of the article
}

region is fused to the gene encoding virus capsid protein, VP1. In the genomes of vesi- and noroviruses, the capsid protein is encoded by a separate ORF2, located towards the 3 '-end of the virus genome. For all caliciviruses, the capsid proteins are produced from an abundant subgenomic RNA synthesized during virus replication. The same RNA serves as bicistronic template for the expression of a minor capsid protein, VP2. The ORF encoding VP2 is near the 3'-end of the virus genome and is conserved among caliciviruses [5].

The genus Vesivirus currently contains two approved species, Vesicular exanthema of swine virus (VESV) and Feline calicivirus (FCV), and a diverse group of unassigned, phylogenetically-related viruses [6]. There are several wellrecognized animal pathogens among vesiviruses that have been associated with a variety of disease conditions. These include diarrheal disease in dogs [7], respiratory illness, vesicular lesions, and epidemic hemorrhagic fever in cats [8,9], and vesicular lesions in several other host species including swine, pinnipeds and humans [10-12]. The prototype virus of the genus Vesivirus, VESV, was originally isolated from pigs with clinical signs compatible with those caused by infection with foot-and-mouth disease virus (FMDV)
C Biomed Central

(C) 2012 Sandoval-Jaime et al.; licensee BioMed Central Ltd. This is an Open Access article distributed under the terms of the Creative Commons Attribution License (http://creativecommons.org/licenses/by/2.0), which permits unrestricted use, distribution, and reproduction in any medium, provided the original work is properly cited. 
[11]. In 1972, a virus with morphological and biochemical characteristics indistinguishable from those of VESV was isolated on San Miguel Island, California from sea lions and named San Miguel Sea lion virus (SMSV) $[13,14]$. When the experimental infection of pigs with SMSV resulted in a vesicular disease clinically mimicking FMDV and VESV infection, marine animals including ocean fish were retrospectively implicated to be the original source of VESV outbreaks [13,14]. Since then, VESV, SMSV, and other related caliciviruses have frequently been designated as the "marine vesiviruses." In contrast to FCV, which are considered to have restricted host specificity to cats of the family Felidae, the marine vesiviruses have been described as having an unusually broad host range [15-18].

In 1978-79, sixteen new vesivirus strains were isolated from four poikilothermic species (Aruba Island rattlesnake, Crotalus unicolor, Rock rattlesnake, Crotalus lepidus, Eyelash viper Bothrops schlegeli, Bell's horned frog Ceretophyrs ornate) in a California zoological collection. The sixteen viruses were antigenically related and were not neutralized by the available VESV-like reference sera [19]. The new viruses were proposed as members of a new reptilian caliciviruses (RCV) Crotalus 1 (Cro1) serotype [19]. Sequence analysis of a $453 \mathrm{nt}$ region of the Cro1 polymerase gene provided additional evidence for a new vesivirus group [20]. The Cro1 serotype did not appear to be restricted geographically or temporally, or limited to reptile and amphibian hosts. In 1986-7, vesiviruses neutralized by the Cro1 typing serum were isolated from samples collected from three different marine mammals species (Eumetopias jubatus, Zalophus californianus californianus, and Callorhinus ursinus) along the coast of Oregon and California states [16].
In this study, we determined the full-length genome sequence of Cro1 strain 780032I, isolated from the intestine of a Rock rattlesnake (Crotalus lepidus) housed in the San Diego Zoo in 1978. Comparison of the genome sequence of 780032I with those available in GenBank database shows that this Cro1 virus represents a genetically distinct vesivirus strain within the species VESV.

\section{Results and discussion}

Cro1 virus stocks obtained from snake samples collected during the original outbreak in the San Diego Zoo were examined for their ability to grow in cell culture following decades of storage at $-80^{\circ} \mathrm{C}$. In a preliminary screening, virus sample \#780032I obtained from a Rock rattlesnake (Crotalus lepidus) showed efficient growth in Vero cells (CCL-81, ATCC, Manassas, VA). Strong cytopathic effect (CPE) was observed in the virus infected cell monolayer at 24-48 hours post infection (hpi). Plaque titration with an agarose overlay resulted in the formation of detectable plaques at $48 \mathrm{hpi}$ (Figure 1A). The efficiency of virus replication was examined in a multiple-cycle growth curve time course analysis. Inoculation of Vero cells at multiplicity of infection $(\mathrm{MOI})=0.01$ consistently produced titers of $\sim 10^{8}$ $10^{9} \mathrm{pfu} / \mathrm{ml}$ by 24 hours (Figure $1 \mathrm{~B}$ ). To verify virus identity, four individual virus plaques were collected in agarose plugs and virus RNA was extracted from each of them using the RNeasy Kit (Qiagen, Valencia, CA). Purified RNA was employed for the RT-PCR amplification of the virus subgenomic region followed by direct sequencing of the ORF2. Comparison of the ORF2 nucleotide sequences with those in GenBank (AY772540 and AY772541) confirmed the identity of the 780032I strain as Cro1. No evidence of genetic variation was observed among plaque-purified viruses.

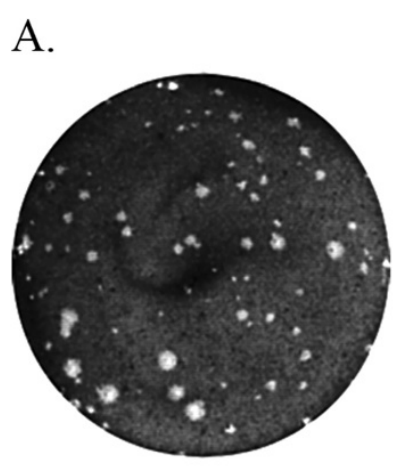

B.

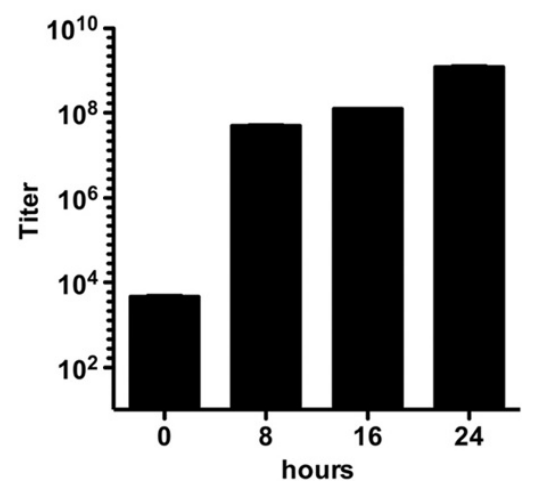

Figure 1 In vitro growth characterization of the $\mathbf{7 8 0 0 3 2}$ strain isolated from Crotalus lepidus. A) The growth of the 7800321 strain results in lysis of infected Vero cells and in the subsequent formation of plaques in a cell monolayer overlayed with $1 \%$ agarose containing growth medium. Plaques can be visualized with crystal violet staining after cells are fixed with formaldehyde. B) The virus titers of the 780032l strain produced by the Vero cells. The cell monolayers were infected with $\mathrm{MOI}=0.01$ and virus titer was measured at different time points by plaque assay. The titers shown are expressed as the mean from two independent experiments performed in duplicate. 
Re-amplified 780032I stock was employed for virus RNA isolation, cDNA amplification and direct sequencing analysis. To determine the full-length genome sequence, several overlapping cDNA fragments of the virus genome were RT-PCR amplified, and sequenced on both strands using a primer-walking technique. The complete genome sequence of the 780032I strain contained $8296 \mathrm{nt}$, excluding the poly (A) tail. Like other vesiviruses, it was comprised of three predicted open reading frames (ORF1, 2, 3) bordered by short 5'- and 3'-end nontranslated (NTR) sequences (Figure 2). The 5 '-end NTR sequence of the 780032I-strain genome was $19 \mathrm{nt}$ in length and was highly conserved among VESVrelated vesiviruses (VESV A48, PAN1, RaV, WCV, SMSV1, v810 and v1415). The 3'-end NTR was $164 \mathrm{nt}$ in length, and showed a higher degree of nucleotide variation when compared to that of other vesiviruses. The lengths of the ORF1, ORF2 and ORF3 were found to be $5643 \mathrm{nt}, 2121 \mathrm{nt}$ and $348 \mathrm{nt}$, respectively. The stop codon of ORF1 (in bold) and the start codon of ORF2 (underlined) were separated by five nucleotides (UAGCCAUUAUG), while ORF2 and ORF3 overlapped by four nucleotides (AUGA) similar to other vesiviruses. The 3'-end of the 780032I ORF1 (nt 5648-5660) contained a conserved sequence motif that showed a high level of identity (10 out of $13 \mathrm{nt}$ ) with the first $13 \mathrm{nt}$ of the virus genome. In addition, this motif was highly similar (9 out of $13 \mathrm{nt}$ ) to the 5 '-end of the FCV subgenomic RNA that was mapped by primer extension analysis [21,22]. Taken together, these data supported the predicted start of the 780032I subgenomic RNA as position 5648 of the genome.
The vesivirus ORF1 encodes a nonstructural polyprotein that undergoes co-translational proteolytic processing during virus replication [23]. A cascade of proteolytic events mediated by the virus-encoded proteinase gives rise to the virus mature nonstructural proteins and their intermediate forms. The Cro1 ORF1 encodes an 1880 amino acid nonstructural polyprotein with a predicted molecular mass of $209 \mathrm{kDa}$. Motif scanning [24,25] and comparative sequence analysis of the Cro1 polyprotein revealed the presence of the characteristic NTPase $\left({ }^{590} \mathrm{GppgcGKT}^{597}\right)$, 3C-like proteinase $\left({ }^{1303} \mathrm{GDCG}^{1306}\right)$ and 3D-like RNAdependent RNA polymerase $\left({ }^{1653}\right.$ GLPSG $^{1657}$ and ${ }^{1701} \mathrm{YGDD}^{1704}$ ) catalytic motifs in an order conserved among caliciviruses. Alignment of the Cro1 ORF1 polyprotein sequence with that of another vesivirus, FCV, that had an experimentally established cleavage map [23] allowed prediction of the putative cleavage sites and, correspondingly, the sizes of mature nonstructural proteins. The predicted Cro1 polyprotein cleavage sites were conserved among VESV-related vesiviruses and, with the exception of cleavage site between NS3 and NS4 proteins, all carried a glutamic residue in the P1 position and alanine, serine or glycine residues at the position P1' (Figure 2). Of interest, the NS3-NS4 scissile bond was predicted between glutamine and alanine residues. The order and sizes of the proteins defined by these cleavage sites were as follows: $16.2 \mathrm{kDa}$ NS1 - 32.2 kDa NS2 - $39.6 \mathrm{kDa} \mathrm{NS}^{\text {NTPase }}$ $31.2 \mathrm{kDa} N S 4-13.5 \mathrm{kDa} N 5^{\mathrm{VPg}}-76.5 \mathrm{kDa}$ NS6-7 ${ }^{\text {ProPol }}$.

The vesivirus ORF2 encodes a precursor of the virus capsid protein that is processed by cleavage during capsid protein maturation. Processing of the FCV capsid precursor at an $E^{124}-\mathrm{A}^{125}$ dipeptide by virus NS6-7 ${ }^{\mathrm{ProPol}}$

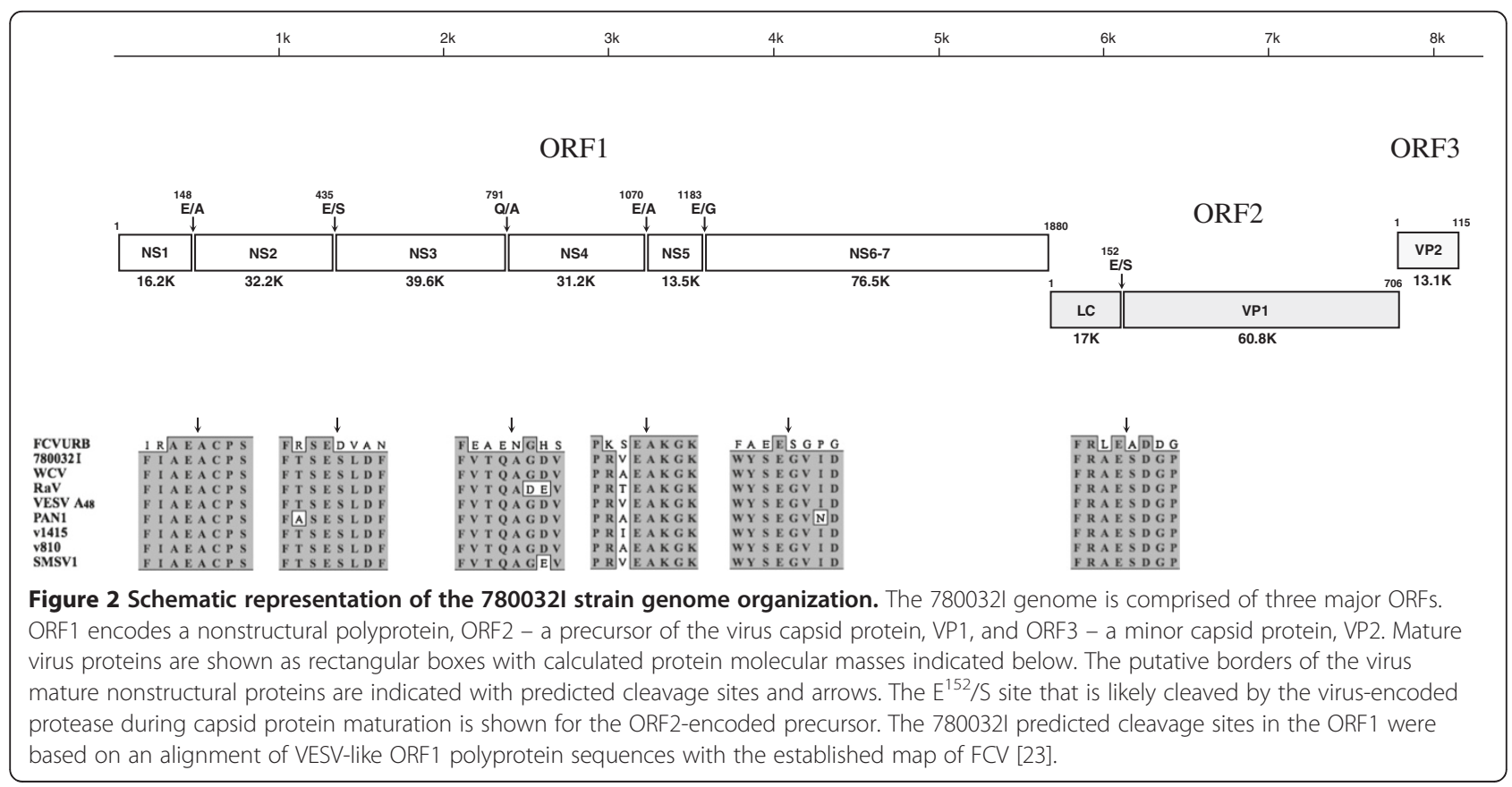


results in removal of an 124 aa-long $\mathrm{N}$-terminal leader sequence (LC) and release of the 59-60 kDa mature form of the virus capsid protein, VP1 [26]. The Cro1 ORF2 sequence is predicted to encode a precursor protein with a molecular mass of $\sim 78 \mathrm{kDa}$. Alignment with the FCV capsid precursor sequence showed the presence of a putative scissile bond between ${ }^{152} \mathrm{E}$ and ${ }^{153} \mathrm{~S}$ (Figure 2). It is likely that, similar to FCV, this cleavage site defines the border between the virus capsid protein leader sequence and mature VP1. Of interest, the site is conserved in the corresponding sequences of the recently characterized v810 and v1415 vesivirus strains isolated from Steller sea lions [27]. The expression of the v810 and v1415 ORF2 sequences that were N-terminally truncated at this site resulted in production and selfassembly of virus-like particles morphologically and antigenically similar to virions [28].

The small ORF3 located at the 3'-end of the vesivirus genome encodes a minor component of virus capsids, the VP2 protein [29]. The protein is expressed from the virus bicistronic subgenomic RNA and has been shown to be essential for the formation of infectious vesivirus virions [30]. The substitution of a $\mathrm{C}$ for a $\mathrm{T}$ at nucleotide 8115 converted a stop codon (TAG) to a glutamine codon (CAG) resulting in a five codon-extension of the ORF3 compared to other VESV-related vesiviruses (data not shown). The 3'end of the calicivirus RNA genome has been shown to play a crucial role in the initiation of virus replication $[31,32]$. Nevertheless, modifications introduced into the genomes of feline calicivirus and murine norovirus showed that this region could tolerate a number of sequence changes while supporting virus replication [30,32].

To elucidate the genetic and phylogenetic relationships of the Cro1 virus with other vesiviruses, multiple sequence alignments of the representative vesivirus genome sequences were generated using the ClustalW algorithm in the Mega5 software package [33]. Comparative genetic distance analysis of the vesivirus sequences revealed the presence of three genetically distinct virus groups, provisionally called here as VESV-like (or marine vesiviruses), FCV-like and CaCV-like viruses. The newly characterized Cro1 virus clustered with the VESV-related group, where differences in sequence identity did not exceed $23 \%$. Pairwise comparison of the Cro1 genome sequence with those of VESV-like viruses showed that the genome of the most distant VESV-like virus, walrus calicivirus, differed from that of Cro1 by $21 \%$. The genetic distances observed between genomes of Cro1 and FCV-like, and $\mathrm{CaCV}$-like viruses were significantly higher at $48 \%$ and $50 \%$, respectively (Table 1 ). Of interest, $\mathrm{FCV}$ and $\mathrm{CaCV}$ were most distant within the genus, with differences in genome sequences reaching 56\% (data not shown).

The genome variability of viruses within the VESV-like group was analyzed using the Plotcon algorithm in the
EMBOSS software package [34]. A similarity profile generated for the multiple sequence alignment of VESV-like viruses revealed the presence of conserved and variable regions across the virus genome (Figure 3). The relatively well conserved sequences included virus nonstructural NS1 and NS4 genes, and the region corresponding to the ORF1-ORF2 junction known to contain a putative transcription start of the virus subgenomic RNA. The VESV-like NS1 and NS4 genes shared an increased level of average nucleotide sequence identity (88.6\%) compared to that of the entire ORF1 (84.6\%). As expected, the most variable part of the virus genome was observed in the ORF2 region that encodes the P domain of the virus capsid protein. The marked variation $(35.4 \%$ nucleotide difference) was consistent with structural and functional studies that have shown that this region of the VP1 is exposed on the surface of the virion and plays a role in receptor binding and immune selection in the host [35]. Of interest, similarity plots for the deduced amino acid sequences encoded by ORF1, 2 and 3, had profiles similar to those of the corresponding nucleotide regions of the virus genome (data not shown).

The profiles of the genome plots generated in a comparison of VESV-, FCV- and CaCV-like vesivirus sequences as well as that for VESV-like sequences alone were similar. Consistent with the generally higher level of genetic divergence existing between vesiviruses from different groups, the similarity indexes calculated for the all-vesivirus sequence alignments were significantly lower. Interestingly, the comparative analysis showed that the NS1 and part of the NS4 sequence were not conserved when compared to the corresponding sequences of the FCV-like and $\mathrm{CaCV}$-like viruses (Figure 3). Furthermore, predicted vesivirus NS1 gene sequences varied in length from 138 to 534 nt between these groups of viruses. For the Cro1, the nucleotide identity of the NS1 gene (predicted to be 148 aa long) was $29.7-38.5 \%$ with the corresponding sequences of $\mathrm{CaCV}$-like viruses, and $51.6-52.2 \%$ with those of FCV-like viruses. The level of similarity for the NS1 deduced amino acid sequences was calculated to be $19.6-22.2 \%$ and $8.8 \%$ between $\mathrm{Cro} 1$ and $\mathrm{CaCV}$-like and between Cro1 and FCV-like viruses, respectively. The function of the calicivirus NS1 protein is unknown and cannot be predicted based on the protein sequence since it does not show significant homology with any established functional sequence motifs. Moreover, the level of similarity of NS1 amino acid sequences between different calicivirus genera does not exceed that of random sequences, precluding identification of conserved sequence motifs.

The observed level of the NS4 sequence identity was significantly lower when the Cro1 sequence was aligned to those of FCV- and $\mathrm{CaCV}$-like viruses. For the latter two, it ranged from 55.2 (Cro1 vs FCV) to $56.4 \%$ (Cro1 
Table 1 Percent nucleotide and amino acid identity of Cro1 strain 780032I with other versiviruses

\begin{tabular}{|c|c|c|c|c|c|c|c|c|c|c|c|c|c|c|c|c|c|c|c|}
\hline \multirow[t]{2}{*}{7800321} & \multirow[t]{2}{*}{ genome } & \multicolumn{2}{|c|}{ NS1 } & \multicolumn{2}{|c|}{ NS2 } & \multicolumn{2}{|c|}{ NS3 } & \multicolumn{2}{|c|}{ NS4 } & \multicolumn{2}{|c|}{ NS5 } & \multicolumn{2}{|c|}{ NS6-7 } & \multicolumn{2}{|r|}{ LC } & \multicolumn{2}{|c|}{ VP1 } & \multicolumn{2}{|c|}{ VP2 } \\
\hline & & nt & aa & nt & aa & nt & aa & nt & aa & nt & aa & nt & aa & nt & aa & nt & aa & nt & aa \\
\hline VESV A48 & 82.9 & 91 & 87.8/92.6 & 83.7 & $90.6 / 96.9$ & 80.2 & $91.3 / 96.6$ & 88.5 & 95/97.1 & 78.5 & $80.5 / 90.3$ & 86.2 & 91.7/96 & 82.7 & 81.6/88.2 & 74.6 & $80.4 / 87.1$ & 80.2 & $86.1 / 93$ \\
\hline PAN1 & 82.5 & 89.2 & $87.2 / 91.2$ & 82.1 & $89.5 / 96.5$ & 79.9 & $91.9 / 96.9$ & 88.1 & 93.6/96.4 & 85 & $95.6 / 98.2$ & 83.9 & $90.8 / 95.6$ & 85.6 & $81.6 / 90.1$ & 75.2 & 82.6/89.6 & 81.6 & $85.2 / 89.6$ \\
\hline v1415 & 82.1 & 84.2 & 79.7/83.1 & 80.3 & $89.2 / 95.8$ & 80.8 & 93.8/98 & 88.3 & $93.5 / 96.8$ & 82.3 & 93.8/99.1 & 82.7 & 93.5/97.6 & 84.2 & 89.5/94.7 & 77 & 84.9/90.5 & 81.3 & $85.2 / 90.4$ \\
\hline v810 & 82.5 & 86 & $80.4 / 87.8$ & 78.4 & $88.5 / 94.8$ & 80 & $92.4 / 97.2$ & 87 & 94.6/97.5 & 83.2 & $95.6 / 100$ & 82.8 & 93.1/97.7 & 87.1 & $89.5 / 94.1$ & 78.9 & $85.3 / 91$ & 84.8 & $89.6 / 92.2$ \\
\hline SMSV1 & 79.2 & 88.3 & 85.8/89.2 & 81.6 & $87.5 / 95.1$ & 80.1 & $90.4 / 96.9$ & 89.5 & $94.3 / 97.1$ & 83.5 & $94.7 / 100$ & 84.4 & $91.5 / 96.4$ & 80.7 & 83.6/89.5 & 64.5 & $67.2 / 80.4$ & 68.4 & $68.7 / 80.9$ \\
\hline WCV & 78.9 & 89 & $85.1 / 90.5$ & 80.6 & $88.9 / 95.8$ & 79.8 & $92.1 / 97.5$ & 87 & $92.1 / 96.1$ & 84.1 & $93.8 / 100$ & 85.9 & $92.4 / 97$ & 81.4 & $82.9 / 90.8$ & 63.9 & $66 / 80.1$ & 67 & 73.9/83.5 \\
\hline RaV & 79.2 & 92.1 & $88.5 / 90.5$ & 82 & $89.5 / 95.8$ & 80.4 & $91.3 / 97.2$ & 86.4 & $92.5 / 95.3$ & 82.9 & $90.3 / 98.2$ & 86 & $93.1 / 97.3$ & 78.3 & 81.6/86.8 & 63.6 & $66.1 / 79.9$ & 65.5 & 71.3/81.7 \\
\hline $\mathrm{CaCV}$ & 49.7 & 29.7 & $7.4 / 22.2$ & 54.1 & $46.9 / 65.4$ & 57.9 & $57.9 / 75$ & 55.2 & $51.9 / 66.6$ & 49.3 & $40.7 / 65.9$ & 58.3 & $56.9 / 71.1$ & 41.6 & $30.6 / 43.1$ & 46.1 & 38.6/52.7 & 32.9 & $29.4 / 40.4$ \\
\hline GCV8 & 51.2 & 38.1 & $6.1 / 19.6$ & 53.1 & $46.2 / 65.1$ & 59.6 & $57.3 / 75.8$ & 55.9 & $52.6 / 67.7$ & 50.9 & $39.8 / 63.4$ & 60.3 & $60.3 / 74.7$ & 45.5 & $27.5 / 40$ & 46.2 & $38 / 54$ & 31.4 & $26.5 / 38.2$ \\
\hline ACV8 & 50.7 & 38.3 & $6.7 / 20.1$ & 53.8 & $46.9 / 65.4$ & 59.5 & $57.6 / 75.8$ & 56.4 & $51.9 / 67.7$ & 52 & $39.8 / 63.4$ & 59 & $60.3 / 74.6$ & 44.1 & 26.9/39.4 & 45.8 & $38.5 / 54.9$ & 31 & $26.5 / 38.2$ \\
\hline ACV9 & 50.5 & 38.5 & $6.7 / 20.1$ & 53.1 & $46.6 / 65.4$ & 59.6 & $57.6 / 75.8$ & 56.2 & $52.3 / 67.7$ & 52 & $39.8 / 63.4$ & 59 & $60.3 / 74.6$ & 43.7 & 26.9/39.4 & 45.8 & $38.5 / 54.7$ & 30.7 & $26.5 / 38.2$ \\
\hline FCVURB & 52.2 & 12.8 & $4.1 / 8.8$ & 55.2 & 48.6/68.1 & 62.2 & $63.2 / 79.8$ & 55.6 & $51.1 / 70$ & 57.3 & $58.8 / 71.1$ & 58.2 & $59.1 / 74.9$ & 32.5 & 19/30.7 & 53.7 & $50.6 / 67.9$ & 41.7 & $20 / 37.4$ \\
\hline FCVF65 & 51.6 & 12.6 & $4.7 / 8.8$ & 54.2 & $45.8 / 65.3$ & 61.6 & $59.8 / 76.7$ & 56.1 & $50 / 68.9$ & 56.1 & $56.1 / 68.4$ & 57.6 & $58.2 / 73.5$ & 30.8 & 19/30.1 & 52.9 & $49.6 / 66.8$ & 40.9 & $20 / 37.4$ \\
\hline FCVF4 & 52.6 & 13.2 & $4.1 / 8.8$ & 53.6 & $47.2 / 67.4$ & 63.9 & $63.8 / 79.5$ & 56.4 & $51.1 / 69.6$ & 56.7 & $57.9 / 69.3$ & 58.8 & $58.7 / 74.6$ & 31.9 & $19.6 / 30.7$ & 54.3 & $51.2 / 67$ & 40.9 & 20.9/38.3 \\
\hline FCVF9 & 51.6 & 13 & $4.1 / 8.8$ & 53 & $47.2 / 66.7$ & 61.5 & $64.3 / 79.5$ & 56.2 & $51.8 / 70.4$ & 54.4 & $56.1 / 71.1$ & 57.9 & $58.8 / 74.9$ & 33 & 18.3/30.1 & 53.7 & $51.2 / 67.7$ & 39.4 & $20 / 36.5$ \\
\hline
\end{tabular}




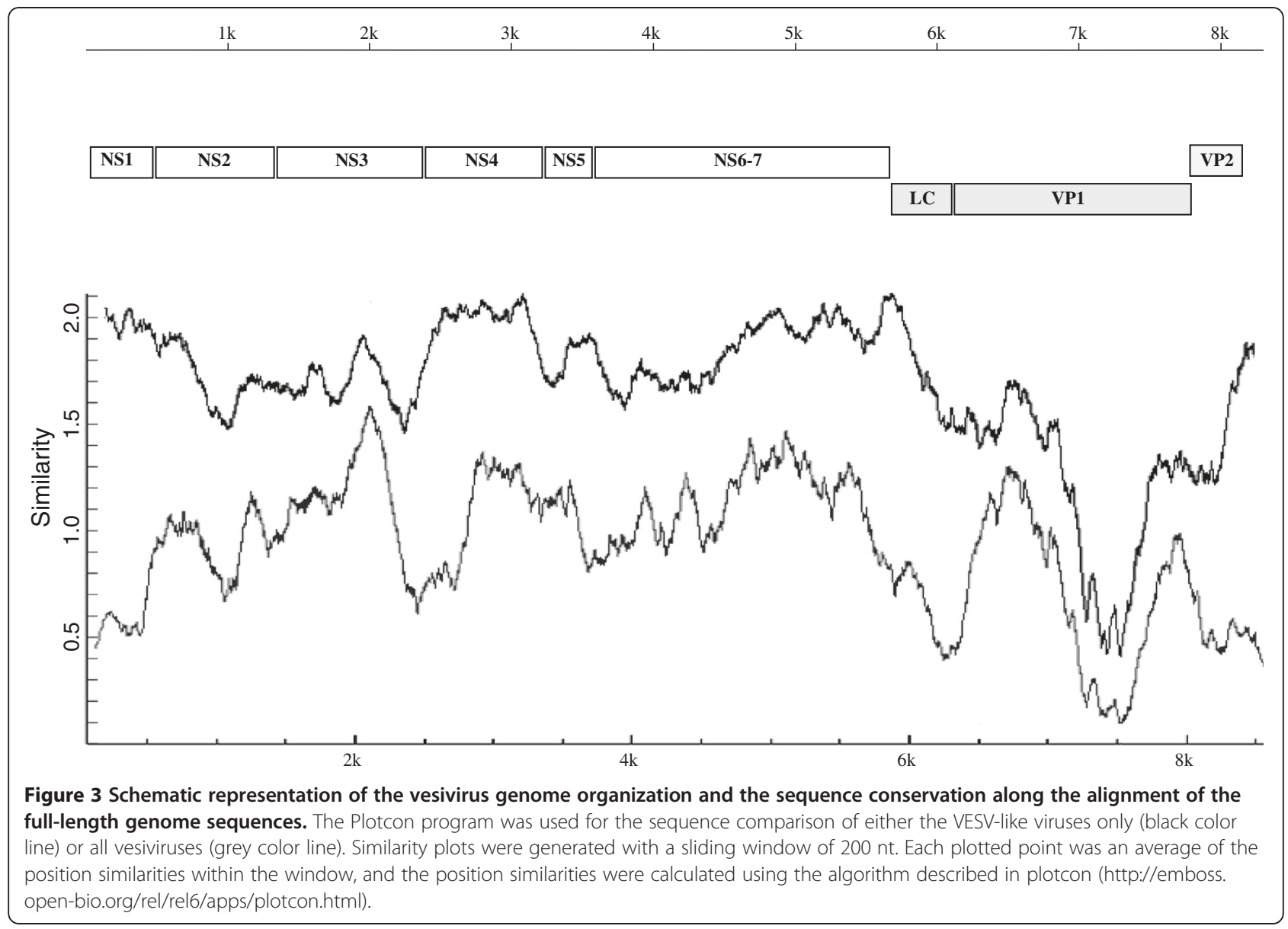

vs $\mathrm{CaCV}$ ). In contrast, the lowest level of sequence identity among VESV-like virus NS4 genes was $86.4 \%$. The similarity profile showed that the most variable sequences of the NS4 gene were located near the 5'-end (Figure 3). Similar to NS1, the NS4 gene is not conserved among viruses from different Caliciviridae genera and represents the second most variable region in the nonstructural ORF of their genomes. Nevertheless, all calicivirus NS4 proteins share a conserved structural feature, which is the presence of a hydrophobic domain. Of interest, a cluster of hydrophobic amino acid residues is located near the C-terminus of the Cro1 NS4 (MacVector's Protein Analysis Toolbox), with amino acids 249-271 predicted to form a membrane-associated helix (TMpred server). Consistent with the putative role of this domain in membrane interactions, biochemical studies showed that transiently expressed NS4 behaved as an integral membrane protein [36]. In addition, different forms of the NS4 were found to localize to the membrane-associated virus replication complexes in calicivirus infected cells $[36,37]$. The significant sequence diversity suggests that NS4 might play an important role in determining the specificity of protein-membrane interactions in the host cell. For example, the subcellular localization of the norovirus NS4 is determined by an ER export signal motif (MERES) conserved only among the noroviruses [38]. Moreover, the presence of the MERES motif was shown to be critical to the NS4 antagonist role in ER/Golgi trafficking [38,39]. Of interest, computational analysis showed that the MERES motif was not present in the Crol sequence. In addition, scanning of the Cro1 NS4 sequence with software designed to identify putative signal and subcellular localization motifs (see Materials and Methods) found no known targeting sequences. The presence of such signals in the Cro1 NS4 protein remains to be established.

Another region of marked sequence variation was observed downstream from the ORF1-ORF2 junction. Plotcon analysis of the vesivirus sequences revealed a low level of nucleotide identity among the virus LC genes, with the lowest identity (30.8\%) between the Cro1 and FCV65 viruses. Accordingly, a lower level of similarity was found for the compared deduced amino acid sequences of this protein, 30.1-30.7\% for FCV-Cro1 and 39.4-43.1\% for CaCV-Cro1 pairs. The function of this protein remains unknown; however, cleavage of the LC from the capsid precursor molecule was crucial for production of infectious virus particles [26]. 
To investigate the phylogenetic relationship of the Cro1 virus with other vesiviruses, a phylogenetic tree was inferred from multiple alignments of representative sequences using the Bayesian method. Figure 4A shows a consensus tree produced by MrBayes3.1.2 for the set of full-length genome sequences. A similar approach was employed to generate additional phylogenetic trees for the vesivirus ORF1 and subgenomic RNA sequences (Figure 4B and 4C). Analysis of the vesivirus genomic tree revealed the presence of three phylogenetic groups. The first group included FCV strains; the second group consisted of viruses related to the $\mathrm{CaCV}$ strain, and the third combined together viruses closely related to the genus prototype strain, VESV A48. A similar topology was observed for the phylogenetic trees inferred for the ORF1 and subgenomic RNA sequences. In all trees, the presence of three major clusters was strongly supported by a high posterior probability value for each clade.

Consistent with genetic distance analysis, the genomic sequence of the Cro1 780032I strain clustered together with those of the VESV-like viruses, making it a member of the marine vesivirus group (Figure 4). Within this cluster, the inferred phylogenetic relationships between RaV, SMSV1, VESV A48, PAN1, WCV and Steller sea lion v810 and v1415 strains were found to be similar to those reported earlier $[17,27,40]$. Of interest, when the phylogenetic tree was inferred for the virus subgenomic RNA sequences, the 780032I strain clustered together with the v810 strain. Similar clustering was observed when a set of the capsid sequences was extended to include two additional sequences available in GenBank, SMSV4 and SMSV17 (accession numbers M87482 and U52005, respectively). In the extended capsid tree, SMSV4 formed a separate group with VESV A48, while SMSV17 clustered with the 780032I, v810 and v1415 strains. However, the latter cluster showed a lack of strong statistical support, with the posterior probability value reaching only 0.68 (data not shown). Of note, the ORF1 and subgenomic sequences of the SMSV1 strain showed inconsistent grouping with those of $\mathrm{RaV}$ and WCV. The observed incongruence of the phylogenetic clustering of these viruses suggested a possible recombinant origin of the SMSV1 strain. Similarity plot analysis of the SMSV1 and other vesivirus genome sequences demonstrated an increased level of nucleotide sequence identity between the subgenomic regions of SMSV1 and $\mathrm{RaV} / \mathrm{WCV}$ strains with a predicted putative recombination site near the junction of the SMSV1 ORF1 and ORF2 sequences (data not shown). However, our preliminary analysis could not identify the second parental strain or its probable lineage (data not shown). Confirmation of a possible recombination event will require further investigation.

More than 40 serotypes of vesiviruses have been identified using serum-neutralization tests [41]. The virus capsid protein VP1 has been shown to encode major antigenic epitopes recognized by antibodies in polyclonal sera of infected animals. Correspondingly,

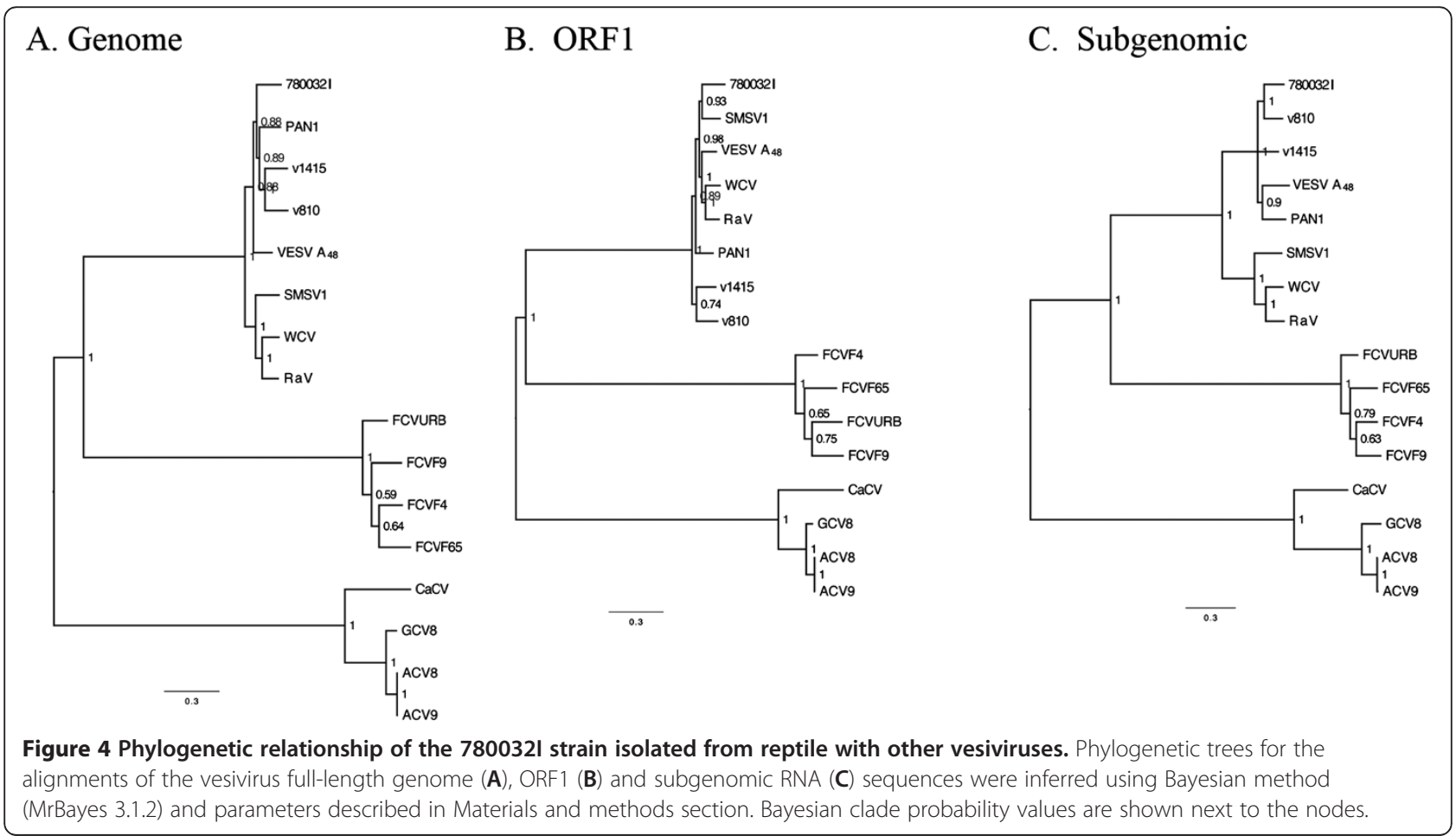


the sequence variability of this protein is thought to provide the molecular basis for serotypic diversity of the vesiviruses. The highest similarity of amino acid sequence (91.4\%) for the Cro1 VP1 protein was observed with the VP1 protein of a recently described v810 strain [28]. Interestingly, most (72 out of 79) of the amino acid changes in the VP1 sequence of these strains mapped to the C-terminal part of the protein, a region of the vesivirus genome with the highest level of variability (Figure 3). Evolutionary conservation scores generated from the corresponding multiple sequence alignment were mapped onto the structure of the SMSV4 VP1 protein with the aid of the ConSurf server software [42]. The X-ray structure of the SMSV4 VP1 (resolved to 3.2- $\AA$ ) showed that the vesivirus capsid protein shared a domain organization similar to that of human noroviruses. The protein contained a short Nterminal arm (aa 10-48) and two domains, shell (S) and protruding (P), formed by its N-terminal (aa 49-209) and C-terminal (aa 210-554) parts, respectively. Based on the structure, the $\mathrm{P}$ domain could be further divided into two subdomains, P1 and P2, with the latter formed by the most distally located part of the protein [35]. The ConSurf analysis of the sequence variability coupled with visualization of the data in Chimera [43] showed that the most divergent parts of the $\mathrm{P}$ domain were localized on the surface of the virus capsid and were represented mainly by the sequence of the P2 subdomain (Figure 5A and 5B). It was noteworthy that 54 out of 72 amino acid differences observed between the VP1 P domains of the v810 and 780032I strains were mapped to the P2 region (Figure 5A). Structurally, the P2 sites that accumulated the majority of the sequence differences between these two viruses were located in five loops exposed on the surface of the virus capsid (Figure 5A and 5B). Surface localization of these loops

A.
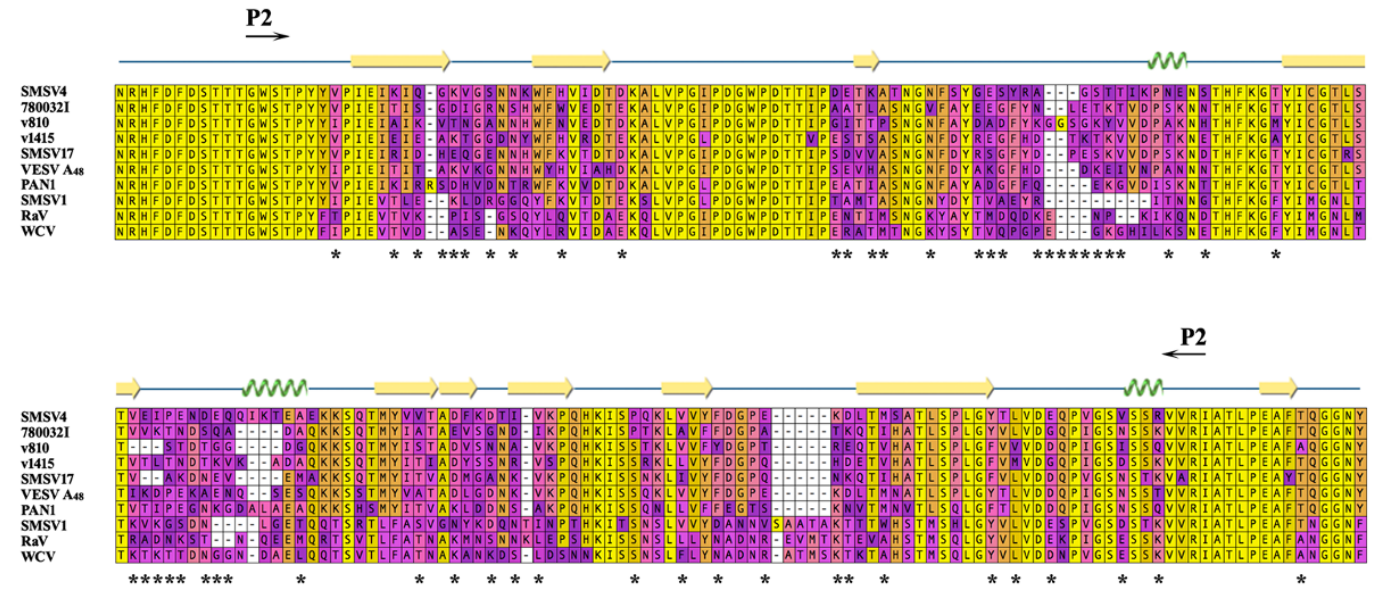

B.
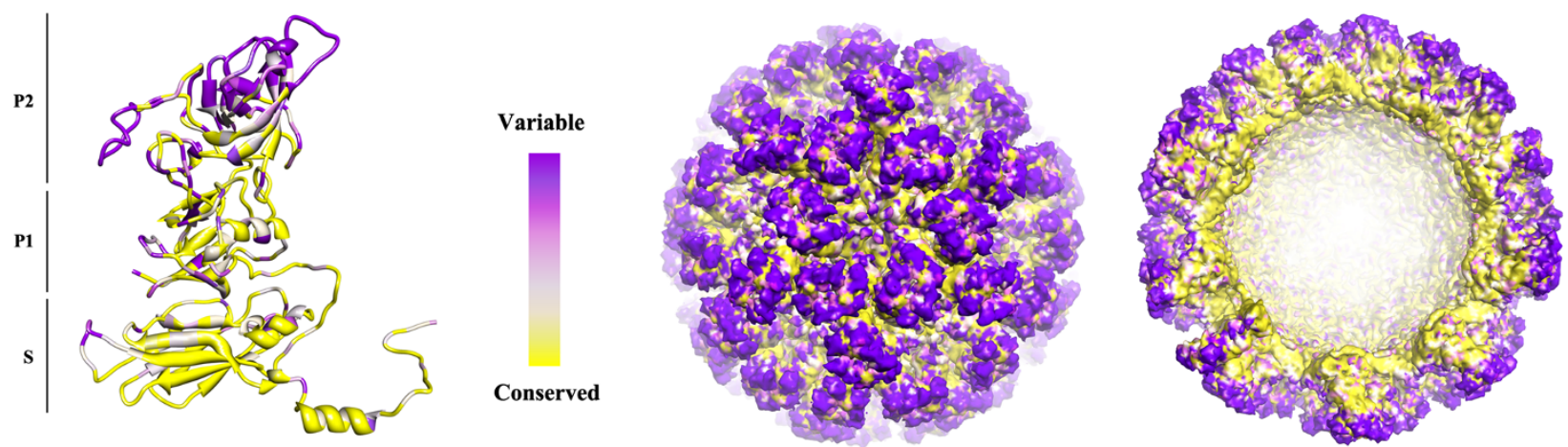

Figure 5 Amino acid sequence variability of the VESV-like virus VP1 proteins. A) Multiple sequence alignment of the VP1 P2 regions of the VESV-like viruses Asterisks indicate positions where differences in amino acid sequences between strains v810 and 7800321 are observed. Structural elements such as $\beta$-strands and a-helices found in the structure of SMSV4 VP1 are depicted above the alignment as hollow box arrows and coils, respectively. B) Ribbon and surface representation of the SMSV4 VP1 protein and its virus capsid assembly inferred using the Chimera program. Color coding of the alignment, ribbon structure and virus capsid were generated using the ConSurf software and is based on variability scale from 0 to $100 \%$, with the most variable residues colored dark purple. 
and their flexibility are thought to play an important role in defining antigenic characteristics of the virus capsid and in its interactions with host cell receptors.

Our finding that Cro1 strain 780032I (isolated from Rock rattlesnake) shares strong sequence identity with the VESV-like marine vesiviruses is consistent with reports that the Cro1 serotype has become established in both aquatic and terrestrial hosts $[16,19]$. The original tissue samples from reptiles in the San Diego Zoo Cro1 outbreak of 1978 are no longer available for analysis, so it is impossible to investigate the outbreak retrospectively. Although the source of this Cro1 virus remains unknown, the continued genetic characterization of known viral isolates in concert with the increasing use of deep sequencing techniques for virus discovery may help track the origin and spread of this and other caliciviruses in nature.

\section{Materials and methods}

\section{Sample isolation and virus amplification}

Reptile tissue samples (small intestine, liver, kidney) were collected in 1978 from hatchling and breeding snakes in the San Diego Zoo. Snakes were experiencing high mortality rates thought to be associated with enteritis and hepatitis of an unknown cause [19]. The tissue samples were homogenized and clarified by low-speed centrifugation. To amplify the virus, the corresponding supernatants were filtered through $0.22 \mu \mathrm{m}$ polysulfone filters, and the resulting filtrates were added to Vero cell monolayers maintained in Eagle's minimal essential medium that was supplemented with penicillin (200 units $/ \mathrm{ml}$ ), streptomycin $(100 \mu \mathrm{g} / \mathrm{ml}), \mathrm{L}$-glutamine $(2 \mathrm{mM})$ and $10 \%$ heat-inactivated fetal bovine serum. The inoculated cells were monitored until the appearance of visible CPE. When CPE in monolayers exceeded 90\%, virus stocks were generated by collection of growth media and low speed clarification of supernatants. After viruses were plaque-purified three times, the amplification procedure was repeated and the clarified supernatants were aliquoted and stored at $-80^{\circ} \mathrm{C}$.

\section{RNA extraction and RT-PCR sequencing}

Viral RNA was extracted from virus stock samples with the RNeasy Mini Kit (Qiagen). The extracted RNA was used as a template for reverse transcription (RT) and PCR amplification of cDNA fragments. The RT-PCR reactions were performed using One-Step RT-PCR Kit (Invitrogen, Carlsbad, CA) and vesivirus genomespecific primer pairs. Briefly, following the initial RT reaction, $30 \mathrm{~min}$ at $45^{\circ} \mathrm{C}$, a denaturation step at $94^{\circ} \mathrm{C}$ was performed for $2 \mathrm{~min}$, followed by 40 cycles of $15 \mathrm{~s}$ at $94^{\circ} \mathrm{C}, 30 \mathrm{~s}$ at $50^{\circ} \mathrm{C}$, and $3 \mathrm{~min}$ at $68^{\circ} \mathrm{C}$. Amplified cDNA fragments were resolved by electrophoresis in $1.0 \%$ agarose gels containing ethidium bromide. The corresponding DNA bands were visualized with UV light and excised from the gel. The DNA was extracted with QIAquick Gel Extraction Kit (Qiagen) and subjected to nucleotide sequencing using the Big Dye Terminator v3.1 Cycle Sequencing Ready Reaction Kit and an automated sequencer, ABI 3100 (Applied Biosystems, Carlsbad, CA). Sequences of the primers that were employed for cDNA fragment amplification are given in Additional file 1: Table S1 and sequences of the primers used for genomic sequencing are available upon request. The sequences of the 5 '-end regions of the virus genome were determined using 5'/3 RACE Kit $2^{\text {nd }}$ Generation (Roche). The corresponding cDNA fragments were synthesized and amplified using 5 '-end sequence-specific and anchor (5'-GACCACGCGTATCGATGTCGACTTTTTTTTTTTTTTTTV-3') primers according to protocol provided by the manufacturer. Following agarose-gel purification, fragments were subjected to direct sequence analysis as described above. Virus genome sequences were assembled using the Sequencher 4.9 program (Genecodes, Ann Arbor, MI).

\section{Computer sequence analysis}

Derived Cro1 nucleotide and amino acid sequences were analyzed, aligned and compared with vesivirus sequences available from the GenBank database using MacVector (MacVector, Inc., Cary, NC), EMBOSS [34] and Mega5 [33] software packages. The GenBank accession numbers for complete vesivirus genome sequences were: Canine calicivirus (CaCV), AB070225; Vesicular exanthema of swine virus (VESV A48), U76874; San Miguel Sea Lion virus-1 (SMSV1), AF181081; Primate calicivirus 1 (Pan1), AF091736; Walrus calicivirus (WCV), AF321298; Rabbit vesivirus (RaV), AJ866991; Feline calicivirus F9 strain (FCVF9), M86379; Feline calicivirus F4 strain (FCVF4), D31836; Feline calicivirus F65 strain (FCVF65), AF109465; Feline calicivirus Urbana strain (FCVURB), L40021; Steller sea lion vesivirus-v810 (v810), EF193004; Steller sea lion vesivirus-v1415 (v1415), EF195384; Calicivirus isolate Allston 2008/US (ACV8), GQ475302; Calicivirus isolate Geel 2008/Belgium (GCV8), GQ475303; Calicivirus isolate Allston 2009/US (ACV9), GQ475301). The genome sequence of the 780032I strain was submitted to GenBank and assigned accession number JX047864.

A plot of average similarity for each set of the aligned vesivirus sequences was generated using the Plotcon program from the EMBOSS software package (http:// emboss.open-bio.org/rel/rel6/apps/plotcon.html).

Scanning for the protein domains and motifs in the predicted protein sequences were performed using InterProScan (http://www.ebi.ac.uk/Tools/pfa/iprscan). Signal peptide and subcellular localization motifs predictions were performed using iPSORT (http://hc.ims.u-tokyo. ac.jp/iPSORT), PSORTII (http://psort.nibb.ac.jp/form2. 
html), SOSUIsignal (http://bp.nuap.nagoya-u.ac.jp/sosui/ sosuisignal/sosuisignal_submit.html), SIG-Pred (http:// bmbpcu36.leeds.ac.uk/prot_analysis/Signal.html), Golgi Predictor (http://ccb.imb.uq.edu.au/golgi/golgi_predictor. shtml), PTS1 predictor (http://mendel.imp.ac.at/mendeljsp/ sat/pts1/PTS1predictor.jsp), Predotar (http://urgi.versailles. inra.fr/predotar/predotar.html), and SignalIP (http:// www.cbs.dtu.dk/services/SignalP). Prediction of the membrane associated domains was performed using TMpred Server (www.ch.embnet.org/software/TMPRED_ form.html).

Bayesian inference of phylogeny was carried out with Mr.Bayes 3.1.2 software [44]. The analysis was performed using Markov chain Monte Carlo sampling under a general time-reversible model of nucleotide substitution with a gamma distribution of rates and a proportion of invariant sites. The search was run for one million generations with tree sampling occurring every 100th generation. Bayesian posterior probabilities and tree topologies were calculated from the consensus of collected tree samples after excluding the first 25\% trees as burn-in. The analyses were performed two times for every set of the sequences. The resulting trees were visualized with FigTree1.3.1 (http://tree.bio.ed.ac. uk/software/figtree/).

\section{Generation of multiple-cycle growth curve}

Vero cell monolayers in six-well plates were infected with the 780032I strain at $\mathrm{MOI}=0.01$, and virus titers were measured at different time points post infection. Briefly, virus stocks were diluted in a supplemented medium (see above), added to cells and allowed to adsorb for one hour at $37^{\circ} \mathrm{C}$. After the incubation, the inocula were removed, the infected cells were washed and fresh growth medium was added to each well. At $0,8,16$, and $24 \mathrm{hpi}$, the plates were frozen and stored at $-80{ }^{\circ} \mathrm{C}$. All collected samples were freeze-thawed three times, and cell debris was removed by low speed centrifugation. Aliquots of the clarified supernatants were used to determine virus titers at each time point by plaque assay [45].

\section{Additional file}

Additional file 1: Table S1. PCR primers used in amplification of the Crol genome cDNA fragments.

\section{Competing interests}

The authors declare that they have no competing interests.

\section{Authors' contributions}

CSJ, KYG and SVS designed the study. CSJ, GIP and SVS performed research and analyzed the data. CSJ, KYG, AWS, SVS wrote the paper. All authors read and approved the final manuscript.

\section{Acknowledgements}

This research was supported by the Intramural Research Program of the $\mathrm{NIH}$, NIAID.

We would like to thank Dr. David Matson at the Eastern Virginia Medical

School, Norfolk, Virginia for providing virus samples.

\section{Author details}

${ }^{1}$ Caliciviruses Section/LID/NIAID/NIH, Bethesda, MD 20892, USA. ${ }^{2}$ Laboratory for Calicivirus Studies, Oregon State University, Corvallis, OR 97330, USA.

Received: 15 August 2012 Accepted: 22 November 2012

Published: 29 November 2012

\section{References}

1. Green KY: Caliciviridae: The Noroviruses, Fields Virology. Volume 1. Philadelphia, PA: Lippincott Williams \& Wilkins; 2007:949-979. [Knipe DM HP (Series Editor).

2. Carstens EB: Ratification vote on taxonomic proposals to the International Committee on Taxonomy of Viruses (2009). Arch Virol 2010, 155:133-146.

3. L'Homme Y, Sansregret R, Plante-Fortier E, Lamontagne AM, Ouardani M, Lacroix G, Simard C: Genomic characterization of swine caliciviruses representing a new genus of Caliciviridae. Virus Genes 2009, 39:66-75.

4. Prasad BV, Matson DO, Smith AW: Three-dimensional structure of calicivirus. J Mol Biol 1994, 240:256-264.

5. Clarke IN, Lambden PR: Organization and expression of calicivirus genes. $J$ Infect Dis 2000, 181(Suppl 2):S309-S316.

6. Green KY: Summary of the first international workshop on human caliciviruses. J Infect Dis 2000, 181(Suppl 2):S252-S253.

7. Matsuura Y, Tohya Y, Nakamura K, Shimojima M, Roerink F, Mochizuki M, Takase K, Akashi H, Sugimura T: Complete nucleotide sequence, genome organization and phylogenic analysis of the canine calicivirus. Virus Genes 2002, 25:67-73.

8. Hoover EA, Kahn DE: Experimentally induced feline calicivirus infection: clinical signs and lesions. J Am Vet Med Assoc 1975, 166:463-468.

9. Radford AD, Coyne KP, Dawson S, Porter CJ, Gaskell RM: Feline calicivirus. Vet Res 2007, 38:319-335.

10. Berry ES, Skilling DE, Barlough JE, Vedros NA, Gage LJ, Smith AW: New marine calicivirus serotype infective for swine. Am J Vet Res 1990, 51:1184-1187

11. Smith AW, Akers TG: Vesicular exanthema of swine. J Am Vet Med Assoc 1976, 169:700-703.

12. Smith AW, Berry ES, Skilling DE, Barlough JE, Poet SE, Berke T, Mead J, Matson DO: In vitro isolation and characterization of a calicivirus causing a vesicular disease of the hands and feet. Clin Infect Dis 1998, 26:434-439

13. Smith AW, Akers TG, Madin SH, Vedros NA: San Miguel sea lion virus isolation, preliminary characterization and relationship to vesicular exanthema of swine virus. Nature 1973, 244:108-110.

14. Smith AW, Skilling DE, Dardiri AH, Latham AB: Calicivirus pathogenic for swine: a new serotype isolated from opaleye Girella nigricans, an ocean fish. Science 1980, 209:940-941.

15. Skilling DE, Barlough JE, Berry ES, Brown RF, Smith AW: First isolation of a calicivirus from the Steller sea lion (Eumetopias jubatus). J Wildl Dis 1987, 23:534-538.

16. Barlough JE, Matson DO, Skilling DE, Berke T, Berry ES, Brown RF, Smith AW: Isolation of reptilian calicivirus Crotalus type 1 from feral pinnipeds. J Wildl Dis 1998, 34:451-456.

17. Ganova-Raeva L, Smith AW, Fields H, Khudyakov Y: New Calicivirus isolated from walrus. Virus Res 2004, 102:207-213.

18. Smith AW: Virus cycles in aquatic mammals, poikilotherms, and invertebrates. In Viral Ecology. Edited by Hurst CJ. Cincinnati, Ohio: Academic Press; 2000.

19. Smith AW, Anderson MP, Skilling DE, Barlough JE, Ensley PK: First isolation of calicivirus from reptiles and amphibians. Am J Vet Res 1986 47:1718-1721.

20. Matson DO, Berke T, Dinulos MB, Poet E, Zhong WM, Dai XM, Jiang X, Golding B, Smith AW: Partial characterization of the genome of nine animal caliciviruses. Arch Virol 1996, 141:2443-2456.

21. Neill JD, Reardon IM, Heinrikson RL: Nucleotide sequence and expression of the capsid protein gene of feline calicivirus. J Virol 1991, 65:5440-5447. 
22. Neill JD: The subgenomic RNA of feline calicivirus is packaged into viral particles during infection. Virus Res 2002, 87:89-93.

23. Sosnovtsev SV, Garfield M, Green KY: Processing map and essential cleavage sites of the nonstructural polyprotein encoded by ORF1 of the feline calicivirus genome. J Virol 2002, 76:7060-7072.

24. Zdobnov EM, Apweiler R: InterProScan-an integration platform for the signature-recognition methods in InterPro. Bioinformatics 2001, 17:847-848.

25. Sigrist CJ, Cerutti L, deCastro E, Langendijk-Genevaux PS, Bulliard V, Bairoch A, Hulo N: PROSITE, a protein domain database for functional characterization and annotation. Nucleic Acids Res 2010, 38:D161-D166.

26. Sosnovtsev SV, Sosnovtseva SA, Green KY: Cleavage of the feline calicivirus capsid precursor is mediated by a virus-encoded proteinase. J Virol 1998, 72:3051-3059.

27. McClenahan SD, Burek KA, Beckmen KB, Knowles NJ, Neill JD, Romero CH: Genomic characterization of novel marine vesiviruses from Steller sea lions (Eumetopias jubatus) from Alaska. Virus Res 2008, 138:26-35.

28. McClenahan SD, Bok K, Sosnovtsev SV, Neill JD, Burek KA, Beckmen KB, Smith AW, Green KY, Romero CH: Expression and self-assembly of virus-like particles from two genotypes of marine vesiviruses and development of an ELISA for the detection of antibodies. Vet Microbiol 2010, 142:184-192.

29. Herbert TP, Brierley I, Brown TD: Detection of the ORF3 polypeptide of feline calicivirus in infected cells and evidence for its expression from a single, functionally bicistronic, subgenomic mRNA. J Gen Virol 1996, 77(Pt 1):123-127.

30. Sosnovtsev SV, Belliot G, Chang KO, Onwudiwe O, Green KY: Feline calicivirus VP2 is essential for the production of infectious virions. J Virol 2005, 79:4012-4024.

31. Simmonds P, Karakasiliotis I, Bailey D, Chaudhry Y, Evans DJ, Goodfellow IG Bioinformatic and functional analysis of RNA secondary structure elements among different genera of human and animal caliciviruses. Nucleic Acids Res 2008, 36:2530-2546.

32. Bailey D, Karakasiliotis I, Vashist S, Chung LM, Rees J, McFadden N, Benson A, Yarovinsky F, Simmonds P, Goodfellow I: Functional analysis of RNA structures present at the 3 ' extremity of the murine norovirus genome: the variable polypyrimidine tract plays a role in viral virulence. J Virol 2010, 84:2859-2870.

33. Tamura K, Peterson D, Peterson N, Stecher G, Nei M, Kumar S: MEGA5: molecular evolutionary genetics analysis using maximum likelihood, evolutionary distance, and maximum parsimony methods. Mol Biol Evol 2011, 28:2731-2739

34. Rice P, Longden I, Bleasby A: EMBOSS: the European Molecular Biology Open Software Suite. Trends Genet 2000, 16:276-277.

35. Chen R, Neill JD, Estes MK, Prasad BV: X-ray structure of a native calicivirus: structural insights into antigenic diversity and host specificity. Proc Natl Acad Sci USA 2006, 103:8048-8053.

36. Bailey D, Kaiser WJ, Hollinshead M, Moffat K, Chaudhry Y, Wileman T, Sosnovtsev SV, Goodfellow IG: Feline calicivirus p32, p39 and p30 proteins localize to the endoplasmic reticulum to initiate replication complex formation. J Gen Virol 2010, 91:739-749.

37. Green KY, Mory A, Fogg MH, Weisberg A, Belliot G, Wagner M, Mitra T, Ehrenfeld E, Cameron CE, Sosnovtsev SV: Isolation of enzymatically active replication complexes from feline calicivirus-infected cells. J Virol 2002, 76:8582-8595

38. Sharp TM, Guix S, Katayama K, Crawford SE, Estes MK: Inhibition of cellular protein secretion by norwalk virus nonstructural protein $\mathrm{p} 22$ requires a mimic of an endoplasmic reticulum export signal. PLoS One 2010, 5:e13130.

39. Sharp TM, Crawford SE, Ajami NJ, Neill F, Atmar RL, Katayama K, Utama B, Estes MK: Secretory pathway antagonism by calicivirus homologues of Norwalk virus nonstructural protein p22 is restricted to noroviruses. Virol J 2012, 9:181.

40. Martin-Alonso JM, Skilling DE, Gonzalez-Molleda A, del Barrio G, Machin A, Keefer NK, Matson DO, Iversen PL, Smith AW, Parra F: Isolation and characterization of a new Vesivirus from rabbits. Virology 2005 , 337:373-383.

41. Smith AW, Boyt PM: Caliciviruses of ocean origin. J Zoo Wildl Med 1990, 21:3-23.
42. Landau M, Mayrose I, Rosenberg Y, Glaser F, Martz E, Pupko T, Ben-Tal N: ConSurf 2005: the projection of evolutionary conservation scores of residues on protein structures. Nucleic Acids Res 2005, 33:W299-W302.

43. Pettersen EF, Goddard TD, Huang CC, Couch GS, Greenblatt DM, Meng EC Ferrin TE: UCSF Chimera-a visualization system for exploratory research and analysis. J Comput Chem 2004, 25:1605-1612.

44. Huelsenbeck JP, Ronquist F: MRBAYES: Bayesian inference of phylogenetic trees. Bioinformatics 2001, 17:754-755.

45. Bidawid S, Malik N, Adegbunrin O, Sattar SA, Farber JM: A feline kidney cell line-based plaque assay for feline calicivirus, a surrogate for Norwalk virus. J Virol Methods 2003, 107:163-167.

doi:10.1186/1743-422X-9-297

Cite this article as: Sandoval-Jaime et al:: Genetic characterization of a reptilian calicivirus (Cro1). Virology Journal 2012 9:297.

\section{Submit your next manuscript to BioMed Central and take full advantage of:}

- Convenient online submission

- Thorough peer review

- No space constraints or color figure charges

- Immediate publication on acceptance

- Inclusion in PubMed, CAS, Scopus and Google Scholar

- Research which is freely available for redistribution

Submit your manuscript at www.biomedcentral.com/submit
C) BioMed Central 